\title{
Rôle inattendu de Scl dans l'endothélium embryonnaire
}

\author{
Amélie Montel-Hagen ${ }^{1}$, Ben Van Handel ${ }^{2}$, Hanna Mikkola ${ }^{2}$
}

${ }^{2}$ Department of Molecular, Cell and Developmental Biology, University of California, Los Angeles, Biomedical Sciences

Research Building, 615 Charles $\varepsilon$. Young Drive South,

Los Angeles, CA 90095, દ́tats-Unis.

amelie.montel.hagen@gmail.com

\section{Mise en place du système hématopoïétique et concept d'endothélium hémogénique} L'hématopoïèse, ensemble des mécanismes conduisant à la production des cellules sanguines, est assurée tout au long de la vie grâce à une population de cellules souches hématopoïétiques (CSH) générées au cours du développement embryonnaire. Les cellules souches hématopoïétiques qui résident dans la moelle osseuse ont la capacité de s'autorenouveler et de se spécialiser dans toutes les différentes lignées cellulaires du système sanguin (érythrocytaire, myéloïde et lymphoïde). Ces propriétés font des cellules souches hématopoïétiques une cible thérapeutique de choix dans le traitement des maladies du sang.

Au cours du développement, les systèmes hématopoïétique et cardiovasculaire sont les premiers à être établis afin de subvenir aux besoins de l'embryon. Les cellules du sang, du cœur et du système vasculaire dérivent toutes du mésoderme. De façon surprenante, les cellules souches hématopoiétiques ne sont pas les premières cellules hématopoïétiques à apparaître chez l'embryon. En effet, le système hématopoïétique est mis en place en plusieurs étapes distinctes au niveau de sites anatomiques multiples. La première étape démarre au niveau des îlots sanguins du sac vitellin et produit des précurseurs de globules rouges (érythrocytes) dits primitifs. Le sac vitellin assure ensuite la production des cellules progénitrices au double potentiel érythrocytaire et myéloïde. Cette étape sera finalement suivie par la génération des cellules souches hématopoïétiques multipotentes. Ce dernier processus très complexe se produit dans une fenêtre temporelle très précise, et au niveau des tissus extra et intra-embryonnaires, dont la région AGM (région contenant l'aorte, les gonades et le mésonéphros), le sac vitellin et le placenta (Figure 1). Une fois générées, les cellules souches hématopoïétiques migrent vers des sites spécifiques tels que le placenta et le foie fœtal, qui leur procurent le microenvironnement nécessaire pour leur expansion et leur maturation. Elles terminent alors leur parcours en colonisant la moelle osseuse.

L'origine cellulaire des cellules souches hématopoïétiques a longtemps été un sujet de controverse. L'observation d'amas de cellules hématopoïétiques étroitement associées aux cellules endothéliales au niveau du sac vitellin de l'embryon avait permis, dès le début du $x x^{e}$ siècle, l'établissement du concept d'endothélium hémogénique, c'est-à-dire de l'existence de cellules endothéliales spécialisées, donnant naissance aux cellules hématopoïétiques. Le développement récent de nouvelles technologies permettant le suivi de la descendance des cellules, ainsi que l'imagerie sur cellules vivantes, ont permis de visualiser directement l'émergence des cellules souches hématopoiétiques à partir de cellules endothéliales, déterminant ainsi leur origine vasculaire (Figure 1) [1-4, 13]. Cependant, les caractéristiques de ce tissu endothélial unique et les mécanismes à l'origine de la production des progéniteurs hématopoiétiques restent méconnus.

\section{$\mathrm{Scl}$ : un rôle double, indispensable à la spécification hématopoïétique de l'endothélium embryonnaire} Scl/Tall (scl : stem cell leukemia; tal : $T$-cell acute lymphoblastic leukemia) est un facteur de transcription indispensable à l'hématopoïèse. Les embryons de souris déficients en $\mathrm{Scl}\left(\mathrm{Scl}^{-/-}\right)$meurent à 9,5 jours de développement $(\varepsilon 9,5)$, en raison d'une absence complète de cellules

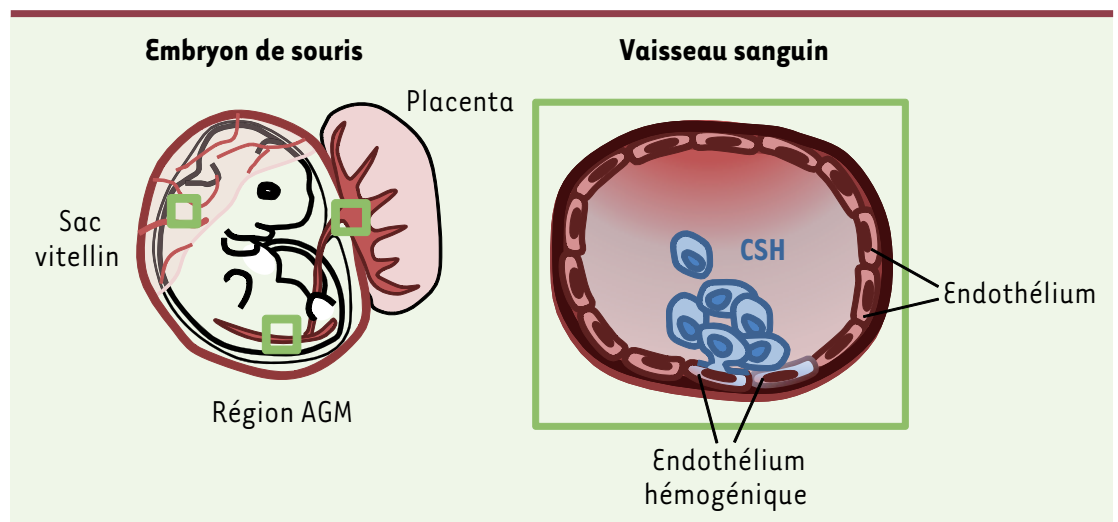

Figure 1. Sites anatomiques d'émergence des cellules souches hématopoïétiques (CSH) et modèle de l'endothélium hémogénique. Au cours du développement, les CSH sont générées au niveau des tissus extra et intra-embryonnaires, tels que la région AGM (région contenant l'aorte, les gonades et le mésonéphros), le sac vitellin et le placenta (gauche). Les nouvelles technologies - suivi de la descendance des cellules, imagerie sur cellules vivantes - ont permis de visualiser directement l'émergence des CSH à partir de cellules endothéliales. L'ensemble de ces cellules vasculaires spécialisées, qui donnent naissance aux CSH, forment l'endothélium hémogénique (droite). 
sanguines et d'un système vasculaire non structuré $[5,6]$. Cependant, les mécanismes utilisés par Scl pour permettre la production des cellules souches et des progéniteurs hématopoïétiques, et particulièrement son mode d'action dans la spécification de l'endothélium hémogénique, sont mal compris.

Nous avons donc étudié le rôle de $\mathrm{Scl}$ dans l'acquisition des propriétés hématopoïétiques de l'endothélium embryonnaire en comparant l'expression des gènes dans les cellules endothéliales de sac vitellin chez des embryons de souris contrôles ou $\mathrm{Scl}^{-/}$. Cette analyse a permis de déterminer le programme génétique régulé par $\mathrm{Scl}$ et a révélé qu'il permettait l'activation de l'ensemble des facteurs de transcription hématopoiétiques au niveau de l'endothélium, conférant à ce dernier ses caractéristiques hémogéniques (Figure 2) [7].
De façon beaucoup plus surprenante, cette étude a montré que $\mathrm{Scl}$ avait également un rôle répressif indispensable. $\varepsilon$ n effet, en l'absence de Scl, les cellules endothéliales expriment de façon anormale un groupe de gènes impliqués dans le développement et la fonction cardiaques. Ces données ont été vérifiées au niveau protéique, et, par exemple, la troponine (une protéine spécifique des cellules cardiaques contractiles ou cardiomyocytes) est exprimée dans le sac vitellin des embryons $\mathrm{Scl}^{-/-}$. De plus, la culture d'explants de sacs vitellins $\mathrm{Scl}^{-/}$a permis d'observer des cellules spontanément contractiles après seulement quatre heures de culture, démontrant ainsi que des cardiomyocytes matures étaient déjà présents dans le sac vitellin (Figure 2) [7]. Afin d'identifier les cellules endothéliales à l'origine des cardiomyocytes en l'absence de Scl, nous avons cherché dans le programme génétique réprimé par $\mathrm{Scl}$ un gène codant pour une protéine de surface qui pourrait caractériser cette population. Pdgfro (plateled-derived growth factor $\alpha$ ), un marqueur du mésoderme cardiogénique [8], était surexprimé de façon significative en l'absence de Scl dans toutes nos analyses. Chez les embryons $\mathrm{Scl}^{-/-}$, nous avons mis en évidence au niveau du sac vitellin, du placenta et de la région AGM (tissus décrits comme hémogéniques) une population de cellules coexprimant le marqueur spécifique des cellules endothéliales CD31 (ou Pecam, platelet endothelial cell adhesion molecule) et le facteur Pdgfra (cellules CD31+ Pdgfr $\alpha^{+}$). L'étude des gènes exprimés par cette population cellulaire particulière a révélé son caractère cardiogénique. De plus, une fois isolées et mises en culture, ces cellules sont capables de générer des cardiomyocytes fonctionnels exprimant la troponine, contenant des sarcomères organisés et capables de

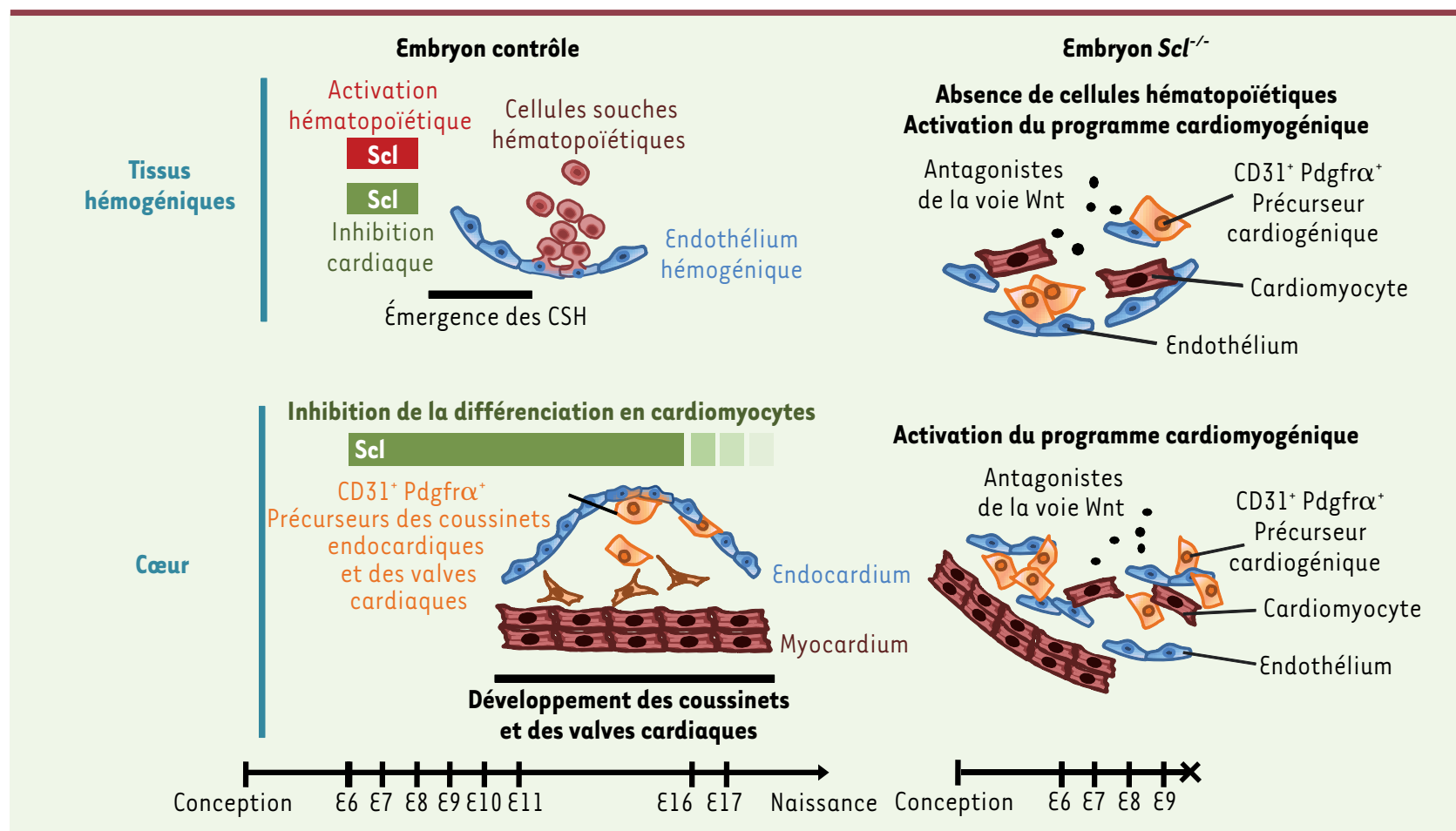

Figure 2. Rôle de Scl au niveau de l'endothélium hémogénique et cardiaque. En conditions physiologiques (gauche), Scl assure la spécification de l'endothélium hémogénique en y activant l'ensemble des facteurs hématopoïétiques (rouge) et en y inhibant le programme cardiaque (vert). Au niveau du cœur, Scl inhibe la différenciation des cellules endothéliales en cardiomyocytes (vert), afin de préserver le développement normal de l'endocardium qui donnera naissance aux coussinets endocardiques et valves cardiaques. En l'absence de Scl (droite), la spécification de l'endothélium embryonnaire est défectueuse et on détecte dans les tissus hématopoïétiques et cardiaques la présence aberrante de cellules progénitrices cardiogéniques $\left(\mathrm{CD}_{3} \mathrm{l}^{+} \mathrm{Pdgfr} \alpha^{+}\right)$et de cardiomyocytes matures. Cette différenciation rapide en cardiomyocytes est liée à une augmentation de l'expression de facteurs antagonistes de la voie canonique Wnt (adapté de [7]). 
battements spontanés. Nous avons donc montré qu'en absence du seul facteur de transcription $\mathrm{Scl}$, les cellules endothéliales des tissus embryonnaires, dont la fonction normale est d'assurer la production et le développement des cellules hématopoïétiques, adoptent les caractéristiques de la lignée cardiaque. Cette fonction répressive de Scl est requise dans les cellules endothéliales de façon intrinsèque et c'est un phénomène limité dans le temps (Figure 2) [7].

\section{Fonction de Scl au niveau cardiaque}

L'absence de Scl ayant pour conséquence la génération de cellules du myocarde dans les tissus hématopoïétiques, nous avons analysé le cœur des embryons $\mathrm{SCl}^{-/-}$afin de mettre en évidence d'éventuelles anomalies du développement de I'endothélium cardiaque ou endocardium. Dans les cavités cardiaques des embryons $\mathrm{SCl}^{-/-}$, l'endocardium est désorganisé, et certaines des cellules endocardiales coexpriment de façon aberrante des marqueurs spécifiques de cellules contractiles du myocarde. Ceci suggère que $\mathrm{Scl}$ intervient dans l'établissement de l'endothélium cardiaque en y réprimant, comme dans les tissus hématopoïétiques, la différenciation en cardiomyocytes. Cependant, au niveau cardiaque, cette fonction répressive de $\mathrm{Scl}$ s'exerce au moins jusqu'à la naissance (Figure 2). De façon surprenante, le cœur est le seul organe dans lequel nous avons pu détecter la population de cellules CD $31^{+}$Pdgfro $^{+}$ chez les embryons contrôles. Leur pourcentage est cependant moindre que dans le cœur des embryons $\mathrm{Scl}^{-/-}$. Nos données suggèrent que cette population, en conditions physiologiques, correspond à des cellules mésenchymateuses, précurseurs des coussinets endocardiques et des valves cardiaques [9], et est donc distincte de la population observée en l'absence de Scl (Figure 2) [7].

\section{Mécanismes impliqués \\ dans la différenciation rapide en cardiomyocytes}

Nous avons étudié le phénotype associé à l'absence de certains gènes régulés par $\mathrm{Scl}$.
Le facteur de transcription Runxl (Runtrelated transcription factor 1 ), qui est indispensable à l'émergence des cellules hématopoïétiques [10,14] et directement activé par $\mathrm{Scl}$, ne semble pas être requis pour réprimer le développement cardiaque [7]. IsIl (islet 1), un facteur de transcription régulant les progéniteurs cardiaques multipotents [11], fait partie des gènes réprimés par Scl. L'absence d'Isll dans les embryons induit une forte diminution des cellules $\mathrm{CD}_{3} 1^{+}$Pdgfr $\alpha^{+}$au niveau du cœur [7]. Nous avons donc analysé des embryons doublement déficients en Scl et Isll pour déterminer si la génération de cellules $\mathrm{CD} \mathrm{I}^{+} \mathrm{Pdgfra}^{+}$en absence de $\mathrm{Scl}$ était aussi dépendante d'Isll. Les doublemutants montrant les mêmes caractéristiques morphologiques et transcriptionnelles que les simple-mutants $\mathrm{Scl}^{-/}$, nos données suggèrent donc que la conversion cardiaque aberrante liée à l'absence de Scl est indépendante d'Is|l [7].

Nous avons également analysé les voies de signalisation en aval de Scl afin de déterminer les mécanismes impliqués dans la différenciation rapide en cardiomyocytes chez les embryons Scl-/-. L'absence de $\mathrm{Scl}$ entraîne une augmentation de l'expression de plusieurs facteurs antagonistes de la voie canonique Wnt (voie impliquée dans l'embryogenèse). Ces mêmes facteurs antagonistes sont connus pour promouvoir la différenciation terminale des cardiomyocytes [12]. Nos résultats suggèrent en effet que les cellules endothéliales déficientes en $\mathrm{Scl}$ sont soumises à une inhibition de la voie canonique Wnt (Figure 2) [7].

$\varepsilon n$ conclusion, l'ensemble de ces données a permis de mettre en évidence une remarquable plasticité au niveau des cellules vasculaires embryonnaires, et d'identifier $\mathrm{Scl}$ comme un facteur clef dans le choix du destin cellulaire de l'endothélium. $\diamond$

An unexpected repressive role for $\mathrm{Scl}$ in the embryonic endothelium

\section{LIENS D'INTÉRÊT}

Les auteurs déclarent n'avoir aucun lien d'intérêt concernant les données publiées dans cet article.

\section{RÉFÉRENCES}

1. Bertrand JY, Chi NC, Santoso B, et al. Haematopoietic stem cells derive directly from aortic endothelium during development. Nature 2010 ; 464 : 108-11.

2. Boisset JC, Van Cappellen W, Andrieu-Soler C, et al. In vivo imaging of haematopoietic cells emerging from the mouse aortic endothelium. Nature 2010; 464 : 116-20.

3. Kissa K, Herbomel P. Blood stem cells emerge from aortic endothelium by a novel type of cell transition. Nature 2010 ; 464 : 112-5.

4. Zovein AC, Hofmann JJ, Lynch M, et al. Fate tracing reveals the endothelial origin of hematopoietic stem cells. Cell Stem Cell $2008 ; 3: 625-36$.

5. Shivdasani RA, MayerEL, Orkin SH. Absence of blood formation in mice lacking the T-cell leukaemia oncoprotein tal-1/SCL. Nature 1995 ; 373 : 432-4.

6. Visvader JE, Fujiwara Y, Orkin SH. Unsuspected role for the T-cell leukemia protein SCL/tal-1 in vascular development. Genes Dev 1998; 12 : 473-9.

7. Van Handel B, Montel-Hagen A, Sasidharan R, et al. $S c l$ represses cardiomyogenesis in prospective hemogenic endothelium and endocardium. Cell 2012 ; $150: 590-605$.

8. Kattman SJ, Witty AD, Gagliardi M, et al. Stagespecific optimization of activin/nodal and BMP signaling promotes cardiac differentiation of mouse and human pluripotent stem cell lines. Cell Stem Cell $2011 ; 8: 228-40$.

9. de Lange FJ, Moorman AF, Anderson RH, et al. Lineage and morphogenetic analysis of the cardiac valves. Circ Res 2004 ; 95 : 645-54.

10. Chen MJ, Yokomizo T, Zeigler BM, et al. Runxl is required for the endothelial to haematopoietic cell transition but not thereafter. Nature $2009 ; 457: 887-91$.

11. Moretti A, Caron L, Nakano A, et al. Multipotent embryonic isll+ progenitor cells lead to cardiac, smooth muscle, and endothelial cell diversification. Cell 2006 ; 127 : 1151-65.

12. Schneider VA, Mercola M. Wnt antagonism initiates cardiogenesis in Xenopus laevis. Genes Dev 2001 ; 15 : 304-15.

13. Boisset JC, Robin C. Origine endothéliale des cellules souches hématopoiétiques : la preuve en image. Med Sci (Paris) $2011 ; 27: 875-81$.

14. Godin I, Cumano A. Les cellules souches hématopoïétiques : une double origine embryonnaire? Med Sci (Paris) 2007 ; 23 : 681-4.
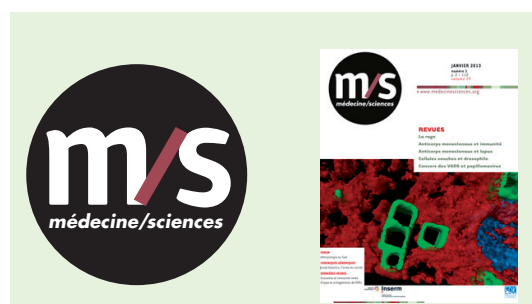

Abonnez-vous à médecine/sciences

Bulletin d'abonnement page 330 dans ce numéro de $\mathrm{m} / \mathrm{s}$ 